\title{
MATERIA ORGANICA EN UNA LAGUNA DE LA COSTA DE SINALOA, MEXICO. (I) : CUANTIFICACION TOTAL
}

GUADALUPE DE LA LANZA E.

Instituto de Biologra, Universidad Nacional Autónoma de México, México

\author{
SYNOPSIS
}

The distribution and variation of total organic matter (TOM) during the rainy season, was studied in the sediments of the coastal lagoon of Huizache-Caimanero, Sinaloa. Two well defined areas werw detected: one with more than 9\% TOM, NE of the lagoon, and other with less than 9\% TOM, SW of the lagoon. An almost homogeneous TOM concentration was found from the surface to $15.20 \mathrm{~cm}$ depth of the sediment core. This is probably due to the continuous addition of TOM from the terrestrial and submerged vegetation.

\section{Introduccion}

Dada la importancia que tiene la materia orgánica en la productividad en los medios acuáticos, se han desarrollado una serie de estudios enfocados a la explotación de los recursos bióticos, que se encuentran directa o indirectamente relacionados con el sustrato sedimentario.

Dentro de las lagunas costeras con que cuenta México, está Huizache y Caimanero, Sin., importante por su recurso pesquero de organismos con hábitos bentodemerzales como lo es el camarón. de gran magnitud económica para el pais. Fscasas han sido las investigaciones realizadas sobre la composición y distribución del material orgánico, de posible consumo por este organismo

El trabajo aquí desarrollado constituye parte de un programa de investigación sobre la composición quimica, procedencia, transformación, utilización y mecanismos de incorporación de la materia orgánica al sedimento, enfocado al conocimiento de la potencialidad del recurso en dicho sistema.

En lo que corresponde a esta contribución, se evalúa el contenido de materia orgánica total, porciento de peso seco (pérdida de agua) y concentración de carbonatos totales en el sedimento, con el objeto de conocer la calidad, cantidad, distribución y los posibles aportes de estos materiales en dicho sustrato

\section{Area de Estudio}

La laguna de Caimanero forma parte del sistema lagunar Huizache y Caimanero, que se encuentra localizada entre $\operatorname{los} 22^{\circ}$ $\mathrm{N}$ y $105^{\circ} 55^{\prime} \mathrm{E}$ de la costa del Pacífico Mexicano. Presenta un clima tropical con dos estaciones al año, la de lluvias que generalmente comienza en julio y termina en octubre y la estación de sequías en que pierde el $78 \%$ de su volúmen (Soto, 1969). Ocasionalmente se encuentra sometida a efectos ciclónicos. Dos principales ríos fluyen al sistema, el Presidio al noroeste y el Baluarte al sureste. Presenta dos comunicaciones hacia el oceáno a través de largos y angostos esteros. Las mareas, según Mendoza von Borstel (1972), adquieren un incremento de nivel a partir de mayo hasta septiembre, para descender a principios de octubre.

En la elección de las localidades de muestreo se consideraron como factores importantes, la batimetría de la laguna y el estado de humectación del medio sedimentario, por lo que los muestreos se decidieron hacer en la estación de lluvias. Para tal efecto se eligieron dos transectos; el que va de Matadero a las Coloradas (A) que incluye las estaciones $1,2,3,4,5$ y 6 y el otro que se inicia de la parte media del anterior hacia el Tapo Pozo la Hacienda (B) con las estaciones $7,8,9,10$ y 11 , ambos muestreados en el mes de septiembre de 1975 y octubre de 1977.

Además, se estudiaron otras localidades dentro de la misma laguna, que representan condiciones extremas como lo son Puente Quemado (estaciones 12 y 13) y el lado este del Tapo Caimanero (estaciones 14,15 y 16), muestreadas en diciembre de 1976 .

\section{Material y Metodos}

Las muestras de sedimento fueron colectadas por medio de nucleadores individuales y seccionadas transversalmente en más - menos $3 \mathrm{~cm}$ de espesor, cada fracción se colocó en cápsulas de porcelana para la determinación de peso seco a $60^{\circ} \mathrm{C}$ durante $12 \mathrm{~h}$. El material seco se pulverizó homogeneizó y cribó en un tamíz de red de $0,50 \mathrm{~mm}$ de abertura. Posteriormente, a cada muestra se le cuantificó la materia orgánica total y carbonatos totales por el análisis térmico diferencial descrito por Dean (1974).

En virtud de que no se trabajó la granulometría de los sedimentos, se estimó adecuado tomar en cuenta el porciento de peso seco (pérdida de agua) con el fin de relacionarlo con el contenido de materia orgánica total.
Los resultados que se muestran en las tabias correspondientes, presentan un orden anual, a pesar de que se analizan por transectos, según las necesidades.

\section{Resultados}

El porciento de peso seco en todas las estaciones estudiadas en septiembre de 1975 y octubre de 1977, presentan en función de la profundidad un ligero aumento que no las diferencía, pero que promediando los valores de cada estación, es posible distinguir dos áreas: la primera que incluye a la mayoría de las estaciones del transecto B y la 6 del A (Figs 1-2 y Tab. 1-3). En forma similar, la segunda área presenta en comparación con la anterior, valores promedio mayores del $50 \%$ en la mayoría del de las estaciones del transecto B y la 6 del A (Figs 1-2 y Tab 1-3). En forma similar, ambas áreas fueron diferenciadas por Gutiérrez (1976) en base a la composición granulométrica, encontrando en términos generales predominancia de material arenoso en la periferia sur de los transectos A y B, y predominio de limo-arcilla en el centro de ambos transectos y parte norte del A.

A pesar de que sólo se realizaron dos transectos de trabajo, las figuras aquí presentadas se desarrollan sólo para hacer significativos los límites entre los valores encontrados.

El contenido de materia orgánica total (MOT) en el sentido vertical, varía escasamente en la mayoría de las estaciones, siendo en ocasiones la misma concentración tanto en la superficie como a la máxima profundidad de muestreo, sin embargo existen algunas estaciones que presentaron disminución en sus secciones más profundas. Según los promedios verticales de cada estación, el contenido de MOT presenta un natrón de distribución horizontal semejante al de porciento de peso seco; esto es, se pueden diterenciar dos áreas; la primer que abarca la mayoría de las estaciones del transecto $\mathrm{A}$ y las 7 y 9 del $\mathrm{B}$ con valores promedio superiores al $9 \%$ de MOT, y la segunda que incluye a la mayoría de las estaciones del transecto $\mathrm{B}$ y la $6 \mathrm{del} \mathrm{A}$, con valores promedio inferiores al $9 \%$ (Figs 3-4 y 1 ab. 1-1II).

Aparentemente existe una relación entre el porciento de peso seco y el contenido de MOT, ya que en la primer área a mayor cantidad de MOT menor porciento de peso seco (mayor humectación), lo que implicaría mayor posibilidad de degradación del material orgánico por su contenido de agua; asi como la segunda área a mayor porciento de peso seco menor contenido de MOT.

La distribución del contenido de carbonatos totales no sigue un patrón bien definido, pero puede observarse que en la mayoría de las estaciones se presenta un aumento preferencial por los últimos centímetros del núcleo muestreado. Por otra parte, promediando los valores de la vertical de cada estación, es posible delimitar las dos áreas ya mencionadas en el porciento de peso seco y MOT (Tab. I y III)

Las estaciones localizadas en Puente Quemado (12 y 13), presentaron los valores más bajos en contenido de MOT, mayor porciento de peso seco y menor contenido en carbonatos totales; las del lado este del Tapo Caimanero $(14,15$ y 16), mayor contenido de MOT, disminución del porciento de peso seco y menor contenido de carbonatos totales. Debido a que la laguna se encuentra sometida a la desecación, probablemente este efecto físico influya en la disminución en el contenido de carbonatos, en ambas localidades (Tab. II).

\section{Discusion}

Ya que la vegetación es uno de los elementos principales en el aporte de material orgánico al sedimento, es fundamental mencionar el tipo de ésta dentro de la laguna; como vegetación sumergida se ha encontrado a Ruppia maritima, Cladophora y En. teromorpha y como colonizadoras cuando la laguna es sometida a la desecación a Salicomia y Suaeda dentro de las más importantes. 


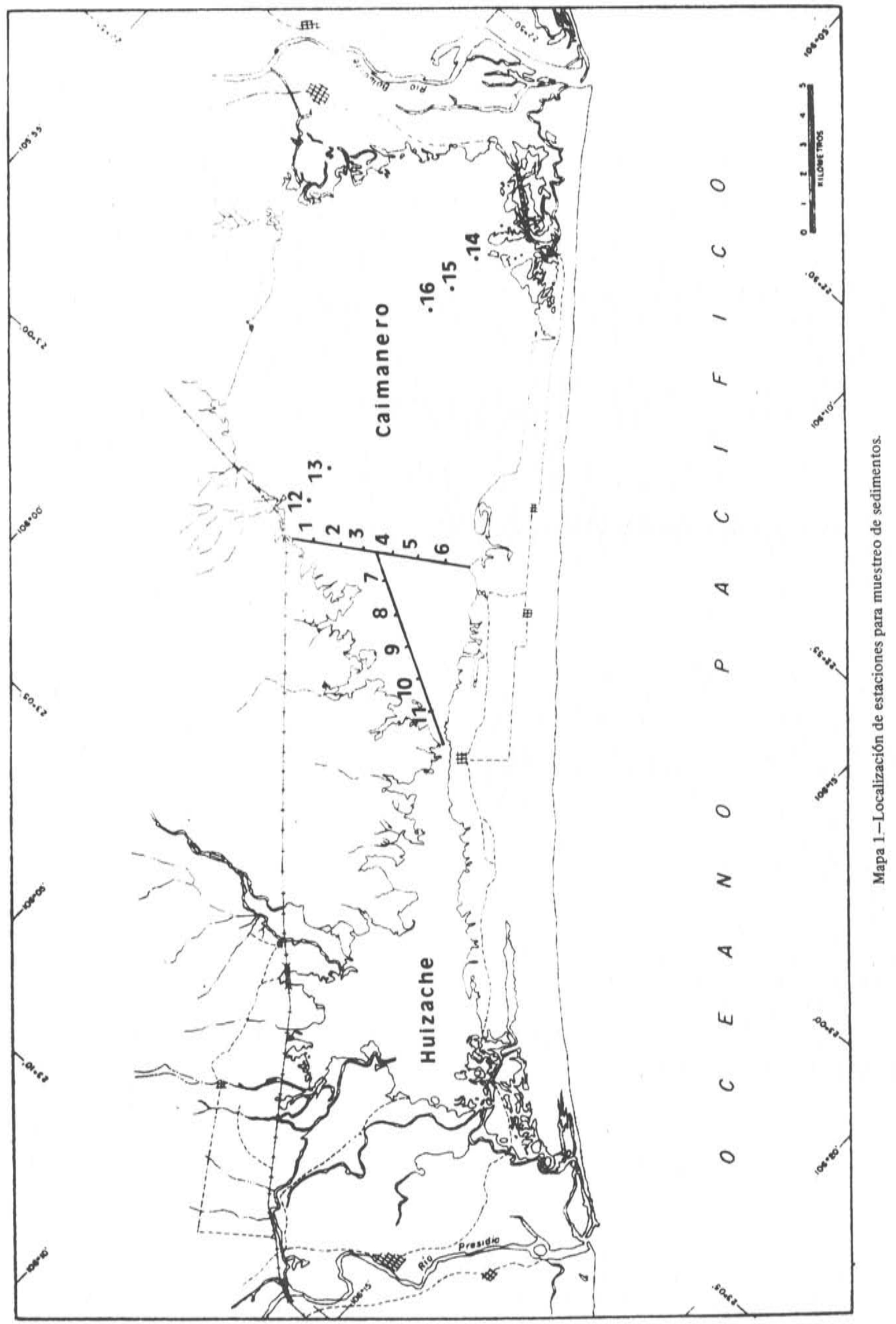




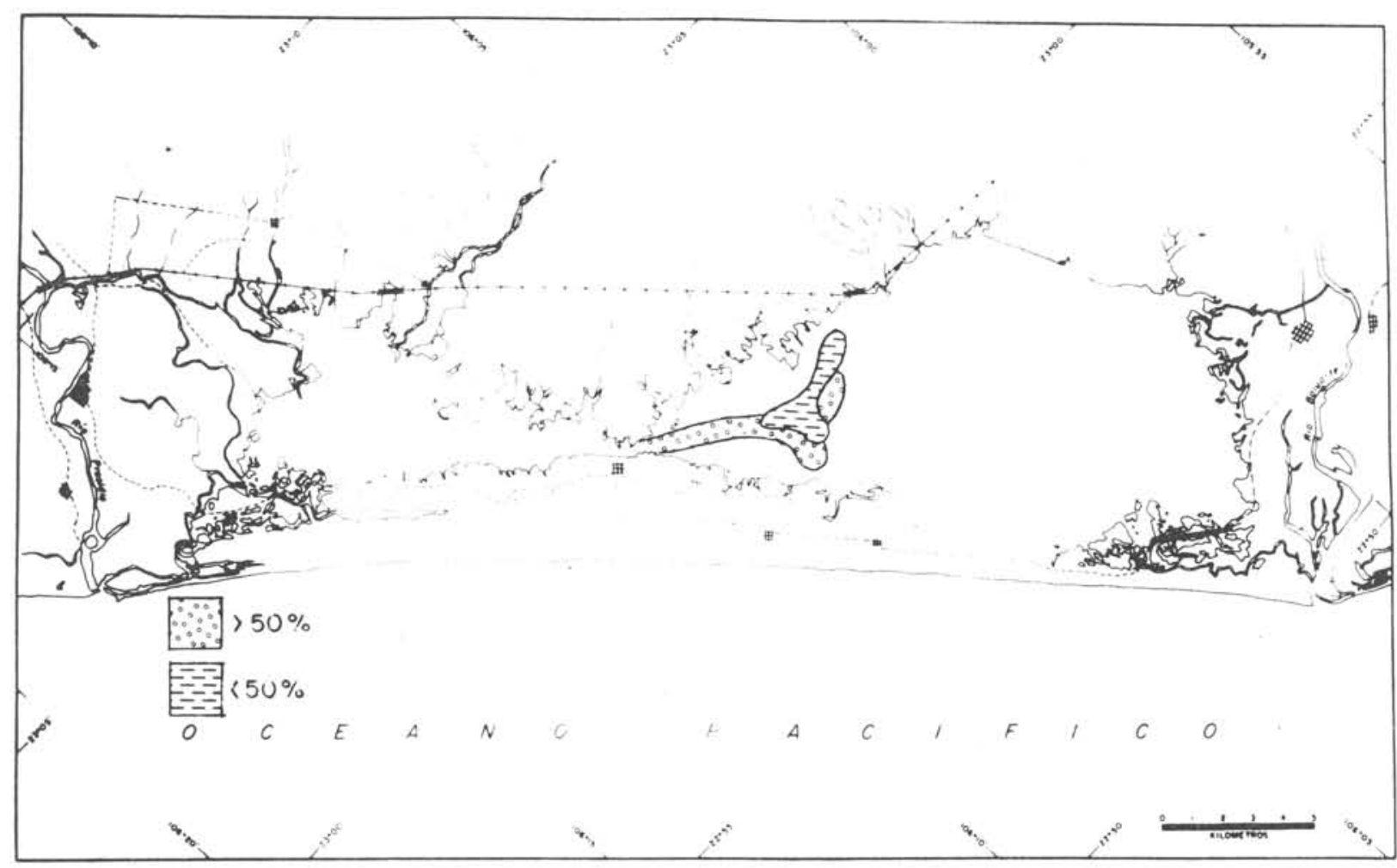

Fig. 1-Distribución de porciento de peso seco respecto al contenido de agua, Septiembre de 1975

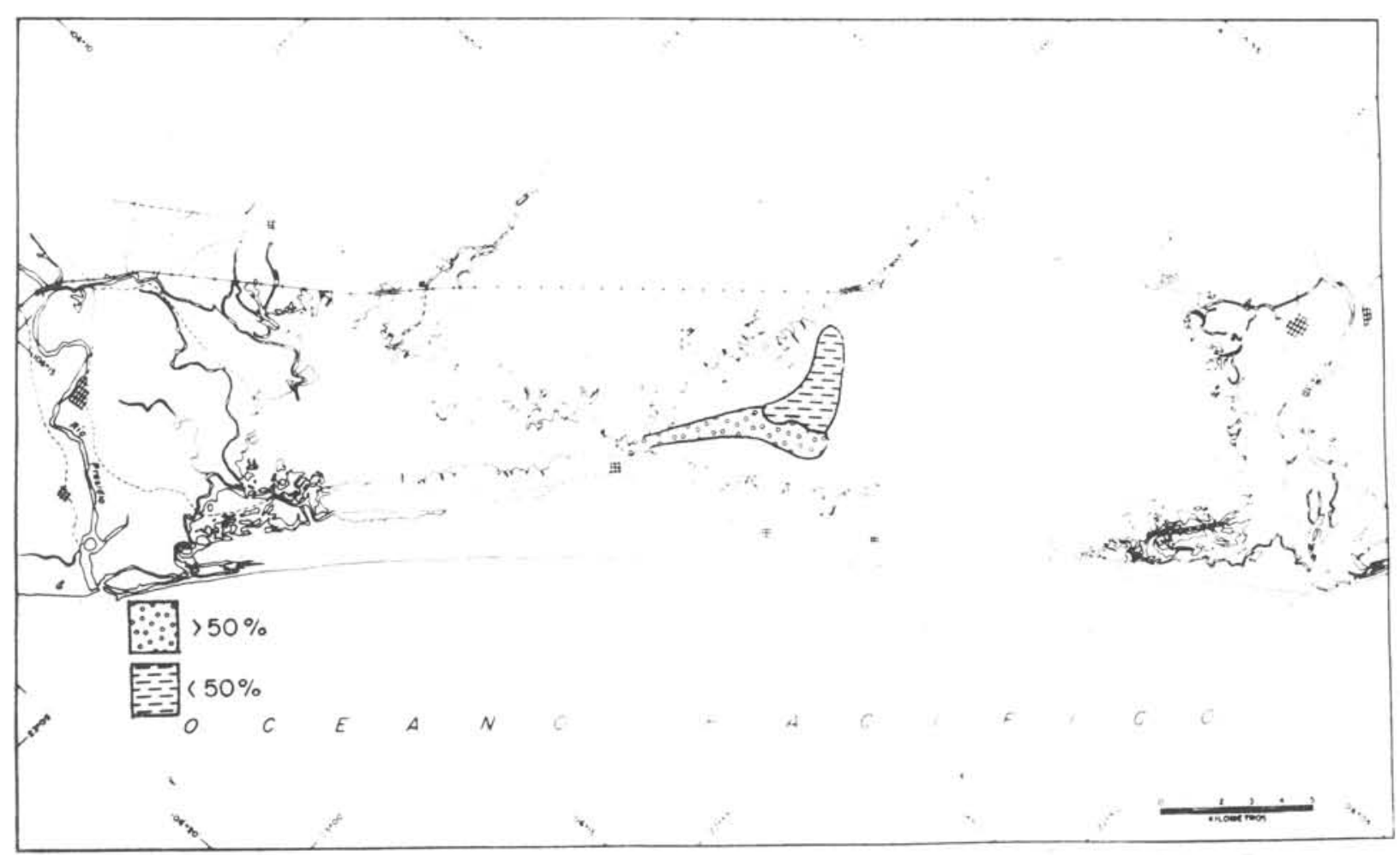

Fig. 2-Distribución de porciento de peso seco respecto al contenido de agua, Octubre 1977 


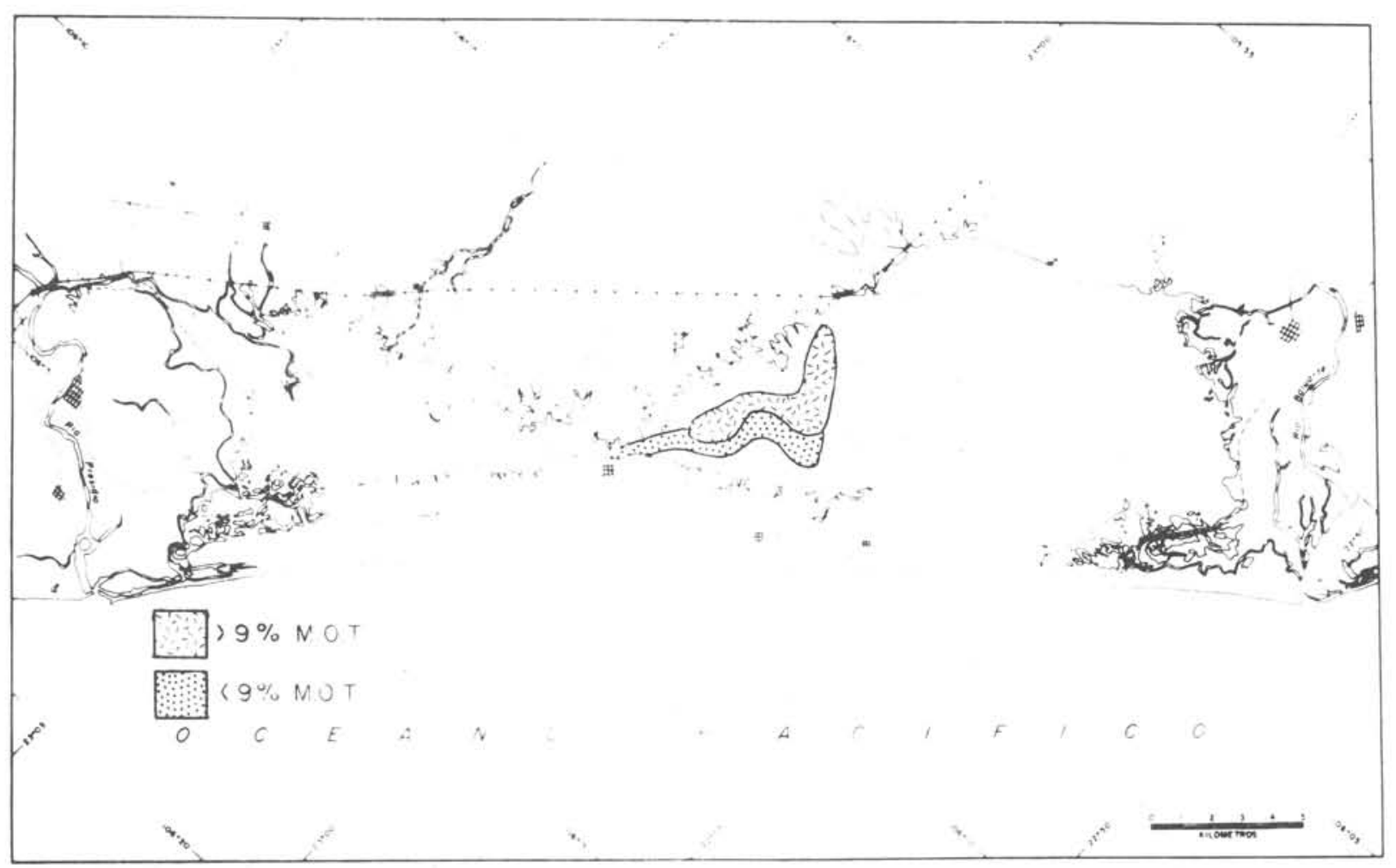

Fig. 3-Distribución del porciento de contenido de materia orgánica total en el sedimento, Septiembre de 1975

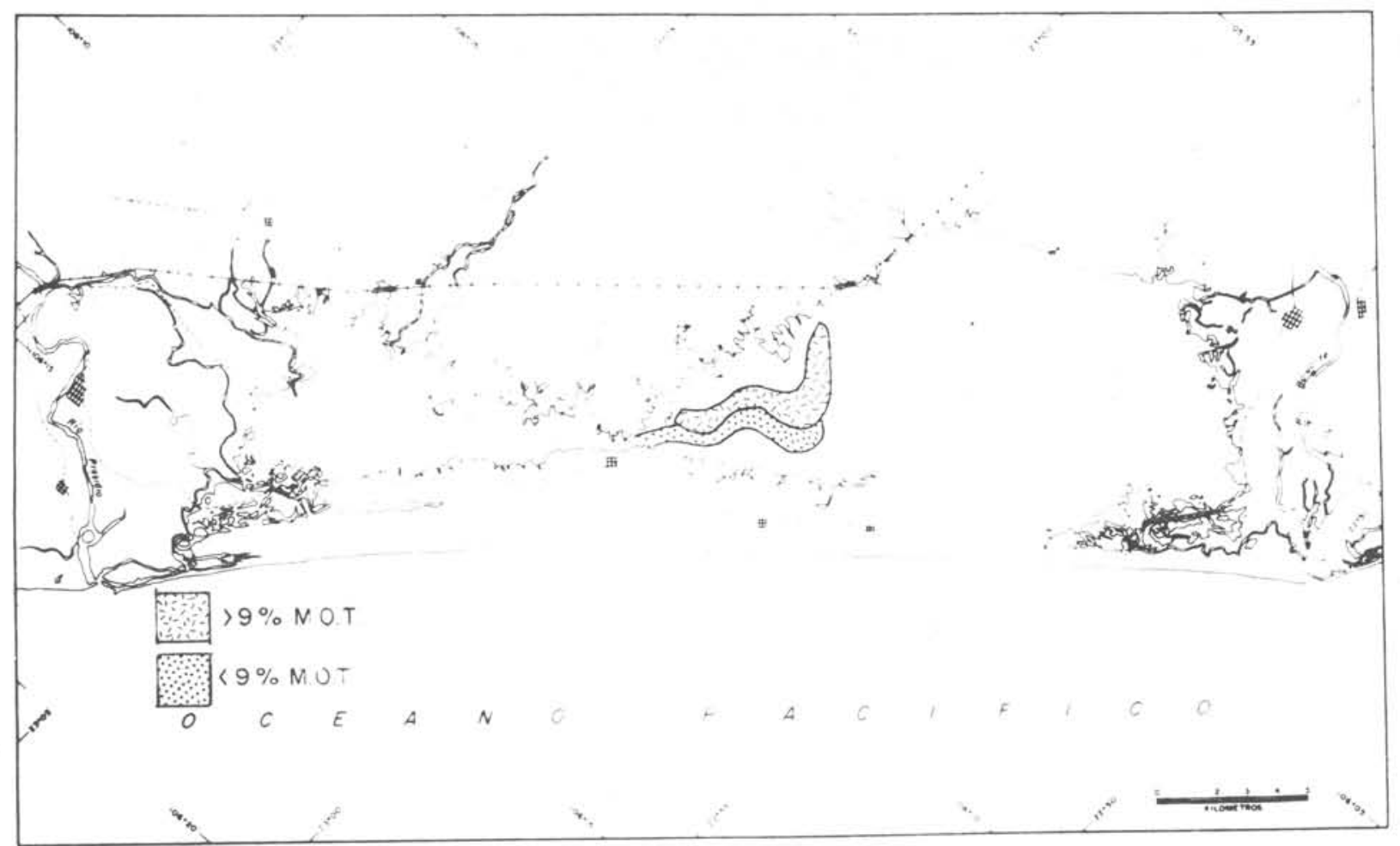

Fig. 4,-Distribución del porciento de contenido de materia orgánica total en el sedimento, Octubre de 1977 
Tabla I

SEPTIEMBRE DE 1975

\begin{tabular}{|c|c|c|c|c|c|c|}
\hline EST. & $\begin{array}{c}\text { * DE PESO } \\
\text { SECO }\end{array}$ & PROM. & $\begin{array}{c}\text { MATERIA } \\
\text { ORG. }\end{array}$ & PROM. & $\mathrm{TOTAL}_{3}$ & PROH \\
\hline 1 & $\begin{array}{l}30.50 \\
41.88 \\
57.62 \\
68.82\end{array}$ & 49.71 & $\begin{array}{r}14.87 \\
13.48 \\
9.41 \\
7.47\end{array}$ & 11.31 & $\begin{array}{l}0.89 \\
1.69 \\
3.78 \\
4.18\end{array}$ & 2.64 \\
\hline 2 & $\begin{array}{l}33.59 \\
35.61 \\
53.24 \\
61.90\end{array}$ & 46.09 & $\begin{array}{r}14.81 \\
14.78 \\
11.02 \\
6.86\end{array}$ & 11.87 & $\begin{array}{l}0.97 \\
0.88 \\
3.65 \\
5.97\end{array}$ & 2.87 \\
\hline 3 & $\begin{array}{l}51.11 \\
58.18 \\
60.47 \\
67.06\end{array}$ & 59.21 & $\begin{array}{r}12.26 \\
10.83 \\
10.04 \\
8.69\end{array}$ & 10.46 & $\begin{array}{l}2.24 \\
1.02 \\
0.93 \\
1.75\end{array}$ & 1.49 \\
\hline 4 & $\begin{array}{l}28.01 \\
55.83 \\
58.81 \\
58.33\end{array}$ & 50.25 & $\begin{array}{r}15.40 \\
9.45 \\
10.42 \\
9.31\end{array}$ & 11.15 & $\begin{array}{l}0.79 \\
4.24 \\
0.85 \\
0.91\end{array}$ & 1.70 \\
\hline 6 & $\begin{array}{l}75.19 \\
75.63 \\
76.46\end{array}$ & 75.76 & $\begin{array}{l}2.16 \\
2.57 \\
2.68\end{array}$ & 2.47 & $\begin{array}{l}0.15 \\
0.18 \\
0.18\end{array}$ & $\begin{array}{ll}0 & 17\end{array}$ \\
\hline 7 & $\begin{array}{l}37.33 \\
41.67 \\
70.16\end{array}$ & 49.72 & $\begin{array}{r}13.30 \\
6.15 \\
4.31\end{array}$ & 7.92 & $\begin{array}{l}0.76 \\
5.00 \\
5.92\end{array}$ & 3.89 \\
\hline 8 & $\begin{array}{l}42.08 \\
54.87 \\
60.06\end{array}$ & 55.67 & $\begin{array}{r}14.49 \\
8.73 \\
9.22\end{array}$ & 11.51 & $\begin{array}{l}0.69 \\
2.17 \\
1.34\end{array}$ & 1.40 \\
\hline 9 & $\begin{array}{l}47.26 \\
70.78 \\
78.66 \\
75.35\end{array}$ & 68.01 & $\begin{array}{r}11.90 \\
6.32 \\
7.86 \\
6.48\end{array}$ & 8.14 & $\begin{array}{l}0.80 \\
0.59 \\
2.13 \\
2.32\end{array}$ & 1.46 \\
\hline 10 & $\begin{array}{l}43.62 \\
62.99 \\
63.22 \\
72.12\end{array}$ & 60.49 & $\begin{array}{l}8.59 \\
6.26 \\
5.43 \\
3.66\end{array}$ & 5.99 & $\begin{array}{l}0.64 \\
2.43 \\
0.32 \\
0.30\end{array}$ & 0.92 \\
\hline
\end{tabular}

TABLA II

DICIEMBRE DE 1976

\begin{tabular}{|c|c|c|c|c|c|c|}
\hline EST. & $\begin{array}{l}\text { DE PESO } \\
\text { SECO }\end{array}$ & PROM. & $\begin{array}{c}\text { MATERIA } \\
\text { ORS. }\end{array}$ & PROM. & $\mathrm{COTALS}^{3}$ & PROY. \\
\hline $\begin{array}{l}\text { Pte. Que } \\
\text { mado } \\
12\end{array}$ & $\begin{array}{l}75.94 \\
81.65 \\
78.82 \\
78.36\end{array}$ & 77.78 & $\begin{array}{l}3.16 \\
3.06 \\
3.09 \\
3.27\end{array}$ & 3.15 & $\begin{array}{l}0.25 \\
0.27 \\
0.25 \\
0.29\end{array}$ & 0.27 \\
\hline 13 & $\begin{array}{l}66.40 \\
69.40 \\
74.72\end{array}$ & 70.17 & $\begin{array}{l}6.54 \\
6.44 \\
4.76\end{array}$ & 5.91 & $\begin{array}{l}0.61 \\
0.39 \\
0.44\end{array}$ & 0.48 \\
\hline $\begin{array}{l}\text { Caimane } \\
\text { ro (Cen } \\
\text { tro No) } \\
14\end{array}$ & $\begin{array}{l}67.36 \\
71.55 \\
70.45\end{array}$ & 69.92 & $\begin{array}{r}10.57 \\
10.37 \\
9.65\end{array}$ & 10.20 & $\begin{array}{l}0.58 \\
0.52 \\
0.87\end{array}$ & 0.66 \\
\hline 15 & $\begin{array}{l}66.04 \\
70.08 \\
87.47\end{array}$ & 74.53 & $\begin{array}{r}9.99 \\
10.21 \\
9.74\end{array}$ & 9.98 & $\begin{array}{l}0.62 \\
0.51 \\
0.62\end{array}$ & 0.58 \\
\hline 16 & $\begin{array}{l}63.56 \\
59.49\end{array}$ & 61.53 & $\begin{array}{l}9.75 \\
9.74\end{array}$ & 9.75 & $\begin{array}{l}0.66 \\
0.66\end{array}$ & 0.66 \\
\hline $\begin{array}{l}\text { Tapo } 1 \mathrm{a} \\
\text { Hacienda } \\
1 \text { ado } \mathrm{Ca} \text {.m. } \\
10\end{array}$ & $\begin{array}{l}63.20 \\
64.69\end{array}$ & 64.01 & $\begin{array}{l}9.04 \\
9.13\end{array}$ & 9.09 & $\begin{array}{l}0.59 \\
0.55\end{array}$ & 0.57 \\
\hline 11 & $\begin{array}{l}58.09 \\
65.46\end{array}$ & 61.78 & $\begin{array}{l}10.94 \\
11.03\end{array}$ & 10.99 & $\begin{array}{l}0.52 \\
0.61\end{array}$ & 0.57 \\
\hline $\begin{array}{l}\text { Tapo Ia } \\
\text { Hacienda }\end{array}$ & & & & & & \\
\hline $\begin{array}{l}\text { 1ado Hui } \\
\text { zache } \\
17\end{array}$ & $\begin{array}{l}65.48 \\
68.92 \\
68.97\end{array}$ & 68.12 & $\begin{array}{r}10.05 \\
9.97 \\
9.87\end{array}$ & 9.96 & $\begin{array}{l}0.51 \\
0.51 \\
0.57\end{array}$ & 0.53 \\
\hline 18 & 68.18 & 68,18 & 11.07 & 11.07 & 0.55 & 0.55 \\
\hline
\end{tabular}


Tabla III

OCTUBRE DE 1977

\begin{tabular}{|c|c|c|c|c|c|c|c|c|c|c|c|c|c|}
\hline Est. & $\begin{array}{c}\% \text { de Peso } \\
\text { Seco }\end{array}$ & Prom. & $\begin{array}{c}\text { Materia } \\
\text { Org. }\end{array}$ & Prom. & $\begin{array}{c}\mathrm{CO}_{3} \\
\text { Totales }\end{array}$ & Prom. & Est. & $\begin{array}{c}\% \text { de Peso } \\
\text { Seco }\end{array}$ & Prom. & $\begin{array}{c}\text { Materia } \\
\text { Org. }\end{array}$ & Prom. & $\begin{array}{c}\mathrm{CO}_{3} \\
\text { Totales }\end{array}$ & Prom. \\
\hline 1 & $\begin{array}{l}37.10 \\
40.09 \\
41.10 \\
41.20\end{array}$ & 39.88 & $\begin{array}{l}12.04 \\
14.40 \\
11.69 \\
11.84\end{array}$ & 12.49 & $\begin{array}{l}0,93 \\
0,89 \\
1.00 \\
1.01\end{array}$ & 0.96 & 7 & $\begin{array}{l}45.86 \\
46.37 \\
48.06 \\
47.46 \\
48.00\end{array}$ & 47.15 & $\begin{array}{l}11.94 \\
11.52 \\
12.07 \\
11.58 \\
11.93\end{array}$ & 11.81 & $\begin{array}{l}0.46 \\
0.48 \\
0.91 \\
1.21 \\
1.37\end{array}$ & 0.89 \\
\hline 2 & $\begin{array}{l}45.17 \\
46.36 \\
46.89 \\
43.71 \\
49.60\end{array}$ & 46.35 & $\begin{array}{r}10.69 \\
11.40 \\
9.74 \\
11.37 \\
10.14\end{array}$ & 10,66 & $\begin{array}{l}1.02 \\
1.01 \\
0.96 \\
1.09 \\
2.49\end{array}$ & 1.31 & 8 & $\begin{array}{l}52.53 \\
61.97 \\
62.38 \\
60.84\end{array}$ & 59.43 & $\begin{array}{l}9.40 \\
6.64 \\
6.60 \\
7.14\end{array}$ & 7.45 & $\begin{array}{l}0.97 \\
2.75 \\
2.39 \\
1.50\end{array}$ & 1.52 \\
\hline 3 & $\begin{array}{l}36.76 \\
40.43 \\
42.69 \\
42.83\end{array}$ & 40.68 & $\begin{array}{l}10.86 \\
10.35 \\
10.54 \\
10.92\end{array}$ & 10.66 & $\begin{array}{l}1.21 \\
0.88 \\
1.04 \\
1.21\end{array}$ & 1.09 & 9 & $\begin{array}{l}41.67 \\
45.90 \\
47.03 \\
51.51 \\
64.30\end{array}$ & 50.08 & $\begin{array}{r}11.51 \\
10.27 \\
11.54 \\
10.20 \\
7.31\end{array}$ & 10.17 & $\begin{array}{l}0.79 \\
0.90 \\
1.02 \\
0.75 \\
0.66\end{array}$ & 0.82 \\
\hline 4 & $\begin{array}{l}36.66 \\
45.32 \\
47.40\end{array}$ & 43.13 & $\begin{array}{r}10.37 \\
9.55 \\
9.61\end{array}$ & 9.84 & $\begin{array}{l}0.77 \\
1.03 \\
0.81\end{array}$ & 0.87 & 10 & $\begin{array}{l}56.23 \\
58.94 \\
61.65\end{array}$ & 60.02 & $\begin{array}{l}8.88 \\
8.49 \\
7.68 \\
8.13\end{array}$ & 8.30 & $\begin{array}{l}0.63 \\
0.72 \\
0.64 \\
0.74\end{array}$ & 0.69 \\
\hline 5 & $\begin{array}{l}44.87 \\
50.83 \\
53.82\end{array}$ & 49.84 & $\begin{array}{l}9.47 \\
8.87 \\
7.62\end{array}$ & 8.65 & $\begin{array}{l}1.52 \\
3.02 \\
3.84\end{array}$ & 2.79 & 11 & $\begin{array}{l}63.24 \\
48.45 \\
50.53\end{array}$ & & $\begin{array}{l}8.13 \\
8.49 \\
8.20\end{array}$ & $\cdot$ & $\begin{array}{l}0.74 \\
0.64 \\
0.66\end{array}$ & \\
\hline 6 & $\begin{array}{l}50.00 \\
50.83 \\
54.95 \\
58.12 \\
\end{array}$ & 53.48 & $\begin{array}{l}7.53 \\
7.74 \\
7.49 \\
6.67 \\
\end{array}$ & 7.36 & $\begin{array}{l}0.84 \\
0.73 \\
0.73 \\
0.79 \\
\end{array}$ & 0.77 & & $\begin{array}{l}52.34 \\
56.83 \\
61.25\end{array}$ & 53.88 & $\begin{array}{l}8.07 \\
8.23 \\
7.28\end{array}$ & 8.05 & $\begin{array}{l}0.78 \\
0.65 \\
0.69\end{array}$ & 0.68 \\
\hline
\end{tabular}

La vegetación sumergida presenta una distribución especial en el sistema lagunar Huizache y Caimanero (Ortega, 1970), que se reflejaría en la concentración de la materia orgánica en el sedimento, sujeta también a la continuidad en el aporte, actividad de organismos (escarbadores) y/o a la tasa de degradación, estas circunstancias son útiles para entender la semejanza y variación en la concentración de MOT en los sedimentos de los transectos estudiados.

Con base en los resultados del contenido de MOT presentados anteriormente, es posible distinguir dos clases de ambientes sedimentarios, uno que representa homogeneidad, que es el de mayor extensión y el otro en el cual la concentración disminuye conforme la profundidad aumenta, de dimensión reducida. En el primer caso, el aporte de MOI proveniente de vegetacion sumergida podría ser continuo y en aquellas estaciones que se encuentran en condiciones extremas de sequedad, el aporte lo substituiría la vegetación halófita terrestre (colonización vegetal alternada), manteniendo asi una fuente constante de material orgánico al sedimento; pero existe la posibilidad de que ésto dependiera de las condiciones ambientales del año anterior, para el óptimo desarrollo de la vegetacion y su imaxumo aporte al seumento. La actuvidad de organismos na sido considerada como uno de los medios para homogeneizar las condiciones físicas y químicas en los primeros centímetros de sedimento, pero como la laguna se ve sometida a condiciones extremas de desecación y aqui no se aboraron esos estudios, no es posible discutirio como un hecho. En el segundo caso, puede especularse que el aporte de MOT no haya sido continuo, que las condiciones ambientales del año anterior no fueron adecuadas o que se presentó una degradación microbiana más - acelerada.

Todo esto se puede justificar en virtud de que la laguna es un medio ambiente muy particular, principalmente debido a los efectos producidos por las condiciones meteorológicas y geológicas de la región.

Entre las características sedimentológicas más importantes y que probablemente se vean reflejadas en la fuente de MOT al sedimento. está el aporte de material sedimentario proveniente de los rios Presidio y Baluarte que durante in estacion lluviosa es muy alto $\mathbf{y}$ asolva con facilidad las comunicaciones con el medio marino. ruesto que ra rasuna es somera, el efecto del viento sobre ella, hace resuspender los sedimentos que posteriormente harán también y... se limite las interacciones mar-laguna (subsidio de energia y entrada de organismos).

Debido a estas condiciones, puede pensarse que la velocidad de depositación de los sedimentos tiene un efecto muy importante en la variación de la concentración de MOT; por lo tanto, si la velocidad de sedimentación es alta, también lo tiane que ser la producción de la materia orgánica y la tasa de degradación no supera al aporte.

Finalmente, es importante hacer notar, que aún cuando la laguna se encuentra en un estado avanzado de senectud, que presenta una significativa tasa de aporte de sedimentos y un considerable grado de azolvamiento en sus comunicaciones que es necesario abrirlas por medio de obras mecánicas y que ha perdido grandes áreas que en la actualidad se utilizan para la agricultura, es un recurso de explotación camaronera muy importante que se asienta en una alta tasa de produtividad primarı local particular y ésta a su vez, en un sistema de ciclos químicos que se realizan en el medio sedimentario donde se encuentra una fuente adecuada de material orgánico, por lo que es necesario realizar un mayor número de estudios tantos químicos, físicos, biológicos, geológicos como ingenieriles tendientes a mantener dicho recurso en explotación.

\section{Referencias bibliográficas}

DEAN Jr., W.E. 1974. Determination of carbonate and organic matter in calcareous sediments rocks by loss on ignition: comparation with other methods. J. sedim. Petrol., 44 (1) : 242-248.

GUTIERREZ, V.G. 1976. Análisis textural de los sedimentos de la Laguna Caimanero, Sin., México. Univ. Nal Autón. de México. (inédito).

MENDOZA VON-BORSTEL, X. 1972. Efectos de la marea en la producción camaronera en lagunas litorales. Mems IV Congr. nac. Oceanogr. (México) : 407-418.

ORTEGA, M.M. 1970. Informe de avance del estudio de vegetación sumergida en los planes piloto Yávaros-Éscuinapa. Informe final de los trabajos contratados en los planes piloto Yávaros-Escuinapa, S.H.R. Inst. Biol. Univ. Nal Autón. de México, : 384-409. 